\title{
XÂY DỰNG GIẢI PHÁP TỐI ƯU VIỆC XÁC ĐỊNH CÁC THAM SỐ CỦA HÀM HIỆP PHƯƠNG SAI LÝ THUYÊTT TRONG PHƯƠNG PHÁP LSC
}

\author{
NGUYẼ̃N THÀNH LÊE(1), NGUYẼ̃N VĂN SÁNG ${ }^{(2)}$, ĐINH XUÂN MẠNH ${ }^{(3)}$ \\ ${ }^{(1)}$ Trường Đại học Lê Quý Đôn; ${ }^{(2)}$ Trường Đại học Mỏ - Địa Chất; \\ ${ }^{(3)}$ Viện Khoa hoc Đo đạc và Bản đồ
}

\section{Tóm tắt:}

Bài báo nghiên cưu về phuơng pháp xác định các tham số tối ưu của hàm hiệp phuoong sai lý thuyết trong phuoong pháp LSC. Co sở lý thuyết của phuơng pháp đã được nghiên cứu chi tiết. Trên co' sở lý thuyết, chương trình "Fitting Covariance Function" xác định các tham số tối ưu của hàm hiệp phuơng sai lý thuyết đã được xây dưng bằng ngôn ngũ C\#. Dựa trên chuơng trình mới xây dụng, nhóm tác giả đã tính toán thục nghiệm cho số liệu thuộc khu vục Miền Trung, các tham số tối ưu nhận được là: $N=224 ; f=0,108 ; A=0,15.10^{6}$; Phwơng sai $=125,91 \mathrm{mGal}^{2} ; R_{E}-R_{B}=-0,471$ $\mathrm{km}$. Các tham số nhận đã được kiểm tra thủ công trên chuơng trình "COVFIT" trong bộ phần mềm GRAVSOFT để khẳng định tính đúng đắn. So với "COVFIT" thì chuơng trình mói có uu điểm là: Tự động tính toán, không cần nhập các tham số đầu vào; Tự động ve đồ thị của hiệp phuơng sai thưc nghiệm và lý thuyết; Tư động lọc ra bộ tham số tối ưu; Ngườ tính không cần có chuyên môn sâu và nhiều kinh nghiệm. Chương trình đã khắc phục được nhũ̃ng hạn chế của chuoong trình "COVFIT".

Tù khóa: Hiệp phương sai thưc nghiệm, hiệp phuoong sai lý thuyết, phưong pháp collocation bình phuơng nhỏ nhất, dị thuờng trọng lực, khớp hàm hiệp phương sai.

\section{1. Đặt vấn đề}

Least-squares collocation (LSC) là phương pháp thường được sử dụng trong các bài toán của Trắc địa, đặc biệt là Trắc địa cao cấp. Việc giải bài toán theo LSC thường được thực hiện dựa trên các bộ phần mềm chuyên dụng. Trong các bộ phần mềm sử dụng, hiện nay, bộ phần mềm GRAVSOFT do nhóm tác giả Forsberg và Tscherning [4] thực hiện được nhiều quốc gia và các nhà nghiên cứu sử dụng. Năm 2014, phương pháp LSC được $\mathrm{Ba}$ Lan sử dụng để xây dựng quasigeoid của mình bằng bộ phần mềm GRAVSOFT [7]. Tại Iran, năm 2019, phương pháp và bộ phần mềm này cũng được sử dụng để xây dựng mô hình goeid quốc gia [6]. Trong [1], các tác giả cũng sử dụng để xác định dị thường trọng lực từ số liệu đo cao vệ tinh. Để giải được bài toán LSC, vấn đề quan trọng là phải xác định được mô hình hiệp phương sai lý thuyết phù hợp nhất với các giá trị hiệp phương sai thực nghiệm. Khi việc làm khớp được thực hiện bằng bộ phần mềm GRAVSOFT thì phải sử dụng chương trình "COVFIT". Chương trình này yêu cầu người dùng thực hiện thử dần các giá trị tham số cho đến khi tìm được giá trị tham số phù hợp nhất. Công việc này đòi hỏi người dùng phải có kiến thức sâu và nhiều kinh nghiệm. Khi sử dụng với mô hình EGM96, mức và hạng lớn nhất chỉ đến 360, thì vấn đề tìm ra các tham số phù hợp tối ưu là không quá phức tạp. Tuy nhiên, với các mô hình thế trọng trường trái đất có mức và hạng bậc cao, $\mathrm{N}_{\max }$ đến 2190 (như EGM2008, EIGEN- 


\section{Nghiên cúu}

6C4) thì để tìm được các tham số phù hợp tối ưu là rất phức tạp và mất nhiều thời gian. Mỗi bộ tham số tìm được, người sử dụng lại phải lưu ra các file riêng biệt, đồ thị biểu diễn độ khớp giữa hiệp phương sai lý thuyết và hiệp phương sai thực nghiệm phải thực hiện thủ công trên phần mềm Excel để xác định được bộ các tham số tối ưu. Vì vậy, vấn đề đặt ra là cần phải xây dựng chương trình làm khớp mới sao cho việc tìm bộ tham số phù hợp tối ưu được thực hiện tự động,

$$
\sum_{i=2}^{N} \hat{\sigma}_{i}\left(\frac{R_{B}^{2}}{r_{P} r_{Q}}\right)^{i+2} P_{i}\left(\cos \psi_{P Q}\right)+\sum_{i=N+1}^{N_{M A X}} \frac{A(i-1)}{(i-2)(i+24)}\left(\frac{R_{B}^{2}}{r_{P} r_{Q}}\right)^{i+2} P_{i}\left(\cos \psi_{P Q}\right)=\operatorname{cov}(P, Q)
$$

Trong công thức $(1), \operatorname{cov}(P, Q)$ là các giá trị của hàm hiệp phương sai thực nghiệm tương ứng giữa hai điểm $\mathrm{P}$ và $\mathrm{Q} ; P_{i}\left(\cos \psi_{P Q}\right)$ là hàm đa thức Legendre ở mức $i ; \psi_{P Q}$ là khoảng cách cầu giữa hai điểm $\mathrm{P}$ và $\mathrm{Q} ; r_{P}$ và $r_{Q}$ là bán kính từ tâm trái đất đến điểm $\mathrm{P}$ và điểm $\mathrm{Q}$; $\hat{\sigma}_{i}$ là sai số phương sai mức và $\mathrm{N}_{\max }$ là mức tối đa của mô hình trọng trường trái đất sử dụng trong tính toán;

Các ẩn cần phải xác định trong công thức (1) là: $\mathrm{N}$ là hệ số mức mở rộng của mô hình trọng trường trái đất $(\mathrm{N}$ có thể nhận giá trị từ 2 đến $\mathrm{N}_{\max }$ ); A là tham số tự do có đơn vị $\left(10 \mu \mathrm{ms}^{-2}\right)^{2}$; $\mathrm{R}_{\mathrm{B}}$ bán kính cầu Bjerhammar (điều kiện $\mathrm{R}_{\mathrm{B}}<$ $\mathrm{R}_{\mathrm{E}}$ ). Vì các sai số phương sai mức của mô hình trường trọng lực trái đất phản ánh trên toàn cầu, trong các tính toán với một khu vực hoặc phạm vi cục bộ, cần phải nhân các sai số phương sai mức với hệ số tỷ lệ $f$. Hệ số tỷ lệ này phải được xác định thông qua quá trình tính lặp. Như vậy, các ẩn số (tham số của hàm hiệp phương sai lý thuyết cần tìm) là: $\mathrm{N}, \mathrm{A}, f, \mathrm{R}_{\mathrm{B}}-\mathrm{R}_{\mathrm{E}}$ (thay cho $\mathrm{R}_{\mathrm{B}}$ ), trong đó $\mathrm{R}_{\mathrm{E}}$ là bán kính trung bình của trái đất.
Úng với mỗi giá trị hiệp phương sai thực nghiệm tính được, ta lập được một phương trình dạng (1). Như vậy, ta sẽ có hệ k phương trình tương ứng với $\mathrm{k}$ giá trị thực nghiệm. Chọn một giá trị của $\mathrm{N}$, giải hệ phương trình trên sẽ tìm được các tham số còn lại. Tham số $f$ được xác định bằng việc giải lặp hệ phương trình với số lần nhất định (thường chọn số lần lặp = 10). Thay đổi $\mathrm{N}$, quá trình giải lại được lặp lại. Không phải $\mathrm{N}$ nào cũng tìm được các tham số $\mathrm{A}, f, \mathrm{R}_{\mathrm{B}}-\mathrm{R}_{\mathrm{E}}$. Có những giá trị của $\mathrm{N}$ làm cho hệ phương trình suy biến.

\subsection{Xây dụng chuơng trình "Fitting} Covariance Function"

Từ cơ sở lý thuyết đã được trình bày chi tiết trong mục 2.1, nhóm tác giả đã thực hiện xây dựng chương trình làm khớp hàm hiệp phương sai lý thuyết với hàm hiệp phương sai thực nghiệm, đồng thời tự động hóa tìm được các tham số của hàm hiệp phương sai lý thuyết phù hợp nhất dựa trên kết quả của bộ tham số có sai số trung phương nhỏ nhất. Chương trình có tên là "Fitting Covariance Function". Chương trình được viết bằng ngôn ngữ $\mathrm{C} \#$. Giao diện của chương trình được thể hiện như Hình 1. 


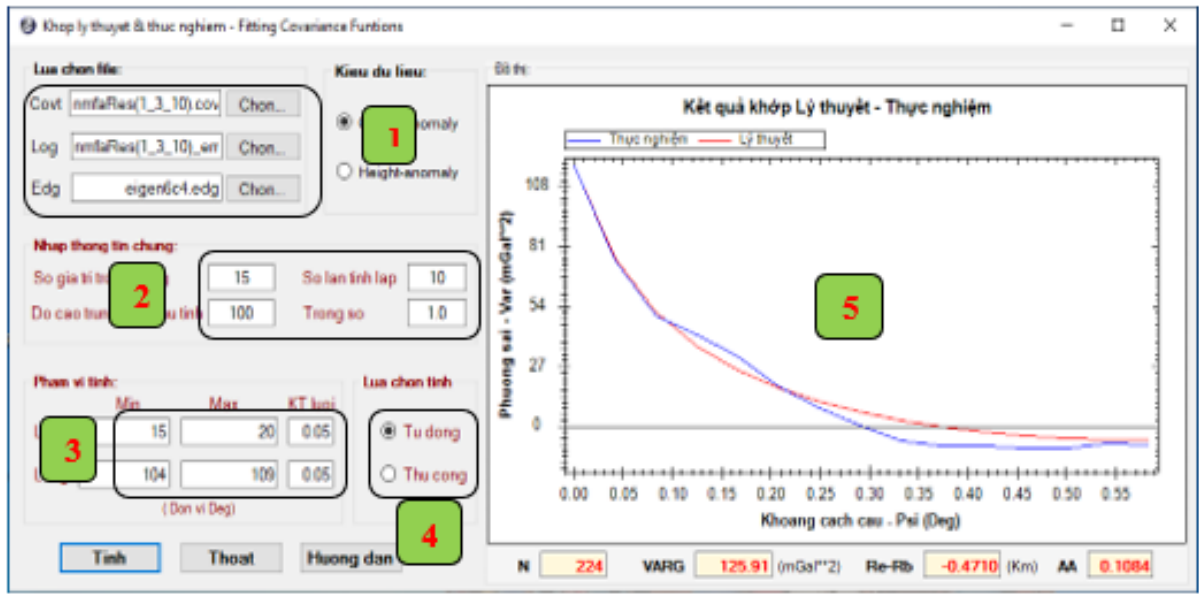

Hình 1: Giao diện chuoong trình "Fitting Covariance Function"

Trong Hình 1:

(1): các file dữ liệu đầu vào của chương trình gồm: file chứa giá trị hàm hiệp phương sai thực nghiệm, file chứa thông tin chung dữ liệu tính và file chứa phương sai mức của mô hình trường trọng lực trái đất;

(2) và (3): thông tin tính toán, được xác định tự động từ các file dữ liệu đầu vào, việc này thuận lợi cho người dùng chưa có kinh nghiệm và đồng thời cũng cho phép thay đổi thông tin trường tính cho phù hợp mục đích tính;

(4): cho phép người tính thực hiện chương trình theo cách tính thủ công hoặc tự động: theo cách thủ công, người tính phải nhập từng giá trị $\mathrm{N}$; còn theo cách tự động, chương trình sẽ thực hiện tính với $\mathrm{N}$ từ 2 đến 2190 và tìm tất cả các bộ tham số thỏa mãn, đồng thời tự động (dựa trên giá trị RMS nhỏ nhất trong các bộ tham số tìm được) lọc lấy bộ tham số có RMS nhỏ phù hợp nhất để thể hiện trên cửa sổ (5).

(5): Cửa sổ biểu diễn kết quả tính. Hiển thị này giúp người tính xem kết quả một cách trực quan.
Khi tính tự động, chương trình sẽ thông báo tổng số bộ tham số tìm được và cho phép người tính xuất kết quả cũng như biểu diễn đồ thị khớp giá trị hàm hiệp phương sai thực nghiệm và lý thuyết trên phần mềm Excel.

\section{Dữ liệu và kết quả tính toán}

\subsection{Khu vục và dũ liệu thử nghiệm}

Giới hạn khu vực thực nghiệm có vĩ độ từ $15^{0} \div 20^{0}$, kinh độ từ $104^{0} \div 109^{0}$, kích thước vùng tính $5^{0}$ x $5^{0}$. Trên đất liền, 9442 trị đo dị thường trọng lực được sử dụng. Dũ liệu dị thường trọng lực trên biển gồm 1430 điểm, mắt lưới $5^{\prime}$ x 5', là số liệu được trích xuất từ mô hình trường trọng lực biển toàn cầu có độ phân giải cao DTU17GRAV [2]. Hình 2 mô tả sự phân bố dữ liệu dị thường trọng lực chân không thuộc phạm vi đất liền và trên biển. Dị thường trọng lực của mô hình EIGEN-6C4 được tính từ trang Web của Trung tâm quốc tế về các mô hình trái đất toàn cầu [5]. Các dữ liệu trọng lực là dị thường trọng lực chân không (Free-air), thuộc hệ tọa độ WGS84, hệ triều là hệ không phụ thuộc triều (tide free). 


\section{Nghiên cúu}

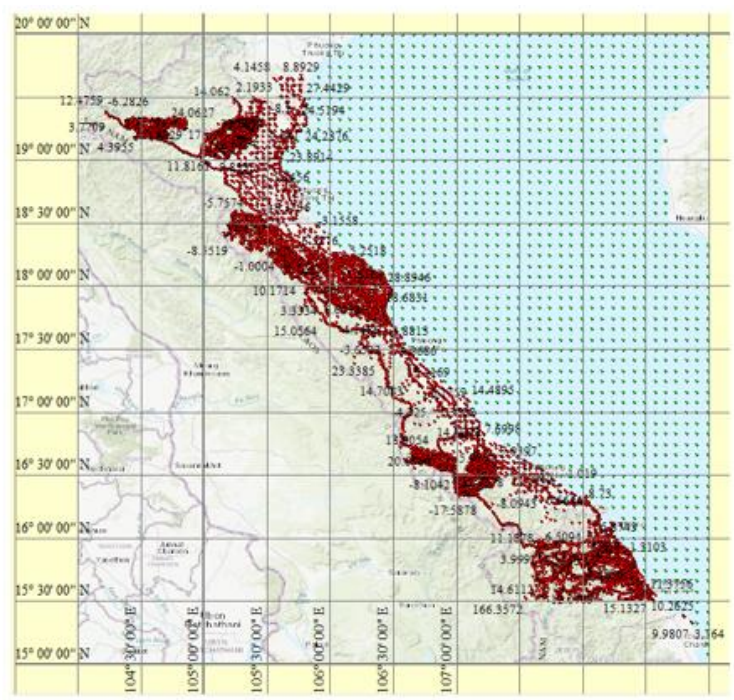

Hình 2: Khu vục thưc nghiệm và dũ liệu tính

-(dị thuoòng trọng lục đất liền; • dị thuoòng trọng lự biển)

\subsection{Kết quả tính}

Dựa trên các giá trị hiệp phương sai thực nghiệm tính từ nguồn số liệu dị thường trọng lực, thực hiện chương trình "Fitting Covariance Function" theo phương pháp tự động với yêu cầu tính lặp 10 lần. Trong kết quả tính, chương trình đã xác định được 63 bộ tham số. Chương trình cũng tự động xác định được bộ tham số tối ưu nhất là: $\mathrm{N}=224 ; f=0,108 ; \mathrm{A}=0,15.10^{6}$; Phương sai $=125,91 \mathrm{mGal}^{2} ; \mathrm{R}_{\mathrm{E}}-\mathrm{R}_{\mathrm{B}}=-0,471 \mathrm{~km}$. Sai số trung phương $\mathrm{RMS}=10,35 \mathrm{mGal}^{2}$. Đồ thị và kết quả tương ứng được thể hiện trong Hình 1 (5). Hiệp phương sai lý thuyết tương ứng với bộ tham số tối ưu và các giá trị của hiệp phương sai thực nghiệm thể hiện trên cột (3) và (2) trong bảng 1 .

Bảng 1: Kết quả đánh giá độ chính xác

\begin{tabular}{|c|c|c|c|c|}
\hline \multirow{2}{*}{ K/c cầu PSI } & \multicolumn{4}{|c|}{ Hiệp phương sai (mGal $\mathbf{~}^{\mathbf{}}$} \\
\cline { 2 - 5 } & Thụ̣c nghiệm & $\begin{array}{c}\text { Fitting Covariance } \\
\text { Function }\end{array}$ & $\begin{array}{c}\text { COVFIT } \\
\text { (GRAVSOFT) }\end{array}$ & Chênh lệch \\
\hline $\mathbf{( 1 )}$ & $\mathbf{( 2 )}$ & $\mathbf{( 3 )}$ & $\mathbf{( 4 )}$ & $\mathbf{( 3 )}-\mathbf{( 4 )}$ \\
\hline 0.000 & 117.9721 & 117.7129 & 117.7129 & 0.0000 \\
\hline 0.042 & 74.5721 & 75.8466 & 75.8466 & 0.0000 \\
\hline 0.083 & 49.6780 & 51.1010 & 51.1010 & 0.0000 \\
\hline 0.125 & 40.7968 & 35.7434 & 35.7434 & 0.0000 \\
\hline 0.167 & 31.0505 & 24.9679 & 24.9679 & 0.0000 \\
\hline 0.208 & 17.6771 & 16.9630 & 16.9630 & 0.0000 \\
\hline 0.250 & 8.0935 & 10.8099 & 10.8099 & 0.0000 \\
\hline 0.292 & -0.1564 & 6.0333 & 6.0333 & 0.0000 \\
\hline 0.333 & -6.6370 & 2.3288 & 2.3288 & 0.0000 \\
\hline 0.375 & -9.1382 & -0.5337 & -0.5337 & 0.0000 \\
\hline 0.417 & -9.2496 & -2.6986 & -2.6986 & 0.0000 \\
\hline 0.458 & -10.3237 & -4.2807 & -4.2807 & 0.0000 \\
\hline 0.500 & -10.2557 & -5.3821 & -5.3821 & 0.0000 \\
\hline 0.542 & -8.0904 & -6.0764 & -6.0764 & 0.0000 \\
\hline 0.583 & -8.6255 & -6.4297 & -6.4297 & 0.0000 \\
\hline
\end{tabular}


Nghiên cúu

\begin{tabular}{|c|c|c|c|c|}
\hline \multirow[b]{2}{*}{ K/c cầu PSI } & \multicolumn{4}{|c|}{ Hiệp phương sai (mGal²) } \\
\hline & Thụ̣c nghiệm & $\begin{array}{c}\text { Fitting Covariance } \\
\text { Function }\end{array}$ & $\begin{array}{c}\text { COVFIT } \\
\text { (GRAVSOFT) }\end{array}$ & Chênh lệch \\
\hline & $\mathbf{N}$ & 224 & 224 & $\mathbf{0}$ \\
\hline \multicolumn{2}{|c|}{ Phương sai } & 125.91 & 125.91 & $\mathbf{0 . 0 0}$ \\
\hline \multicolumn{2}{|c|}{$\mathbf{R}_{\mathrm{E}}-\mathbf{R}_{\mathrm{B}}(\mathbf{k m})$} & -0.471 & -0.471 & 0.000 \\
\hline \multicolumn{2}{|c|}{$\mathbf{A}$} & 179384 & 179400 & -17 \\
\hline \multicolumn{2}{|r|}{ f } & 0.1084 & 0.1084 & 0.0000 \\
\hline \multicolumn{2}{|c|}{ Sai số trung phương } & 10.3472 & 10.3473 & -0.0001 \\
\hline
\end{tabular}

Để khẳng định tính chính xác của chương trình, các tham số của hàm hiệp phương sai lý thuyết được xác định thủ công bằng chương trình "COVFIT" trong bộ phần mềm GRAVSOFT. Kết quả tính toán được trình bày trên cột (4) của bảng 1 . So sánh kết quả tính bằng GRAVSOFT với kết quả tính bằng chương trình tự xây dựng được thể hiện trên cột (3) - (4). Dựa trên kết quả so sánh có nhận xét: Các giá trị tính của hàm hiệp phương sai lý thuyết giữa hai chương trình theo khoảng cách cầu và các tham số $\mathrm{N}$, phương sai, $\mathrm{R}_{\mathrm{E}}-\mathrm{R}_{\mathrm{B}}$, f là giống nhau. Tuy nhiên, sai số trung phương giữa hai chương trình chệnh lệch nhau 0,0001 đơn vị tính; hệ số A lệch nhau giữa hai chương trình 17 đơn vị tính, với hệ số A có giá trị rất lớn, hơn một trăm nghìn đơn vị thì giá trị chênh lệch như vậy là rất nhỏ, không ảnh hưởng đến kết quả tính dị thường độ cao từ số liệu dị thường trọng lực theo LSC. Độ sai lệch xảy ra có thể giải thích do ngôn ngữ lập trình khác nhau và sai số làm tròn trong tính toán. Như vậy, có thể khẳng định là hai chương trình tính là cùng kết quả, đã khẳng định chương trình do nhóm tác giải xây dựng là hoàn toàn chính xác.

Từ thực tế tính toán, các ưu điểm của chương trình do nhóm tác giả xây dựng so với chương trình "COVFIT" của bộ phần mềm GRAVSOFT được thể hiện trong bảng 2 .

Bảng 2: So sánh chuơng trình do nhóm tác giả xây dụng với chuơng trình của COVFIT

\begin{tabular}{|c|l|l|}
\hline TT & $\begin{array}{l}\text { Chương trình COVFIT của bộ phần } \\
\text { mềm GRAVSOFT }\end{array}$ & $\begin{array}{l}\text { Chương trình do nhóm tác giả xây } \\
\text { dựng (Fitting Covariance } \\
\text { Function) }\end{array}$ \\
\hline 1 & $\begin{array}{l}\text { Phải nhập thủ công các tham số đầu } \\
\text { vào phục vụ tính toán. }\end{array}$ & $\begin{array}{l}\text { Tự động tính toán, không cần nhập } \\
\text { các tham số đầu vào. }\end{array}$ \\
\hline 2 & $\begin{array}{l}\text { Không tự động vẽ đồ thị của hiệp } \\
\text { phương sai thực nghiệm và lý thuyết. }\end{array}$ & $\begin{array}{l}\text { Tự động vẽ đồ thị của hiệp phương } \\
\text { sai thực nghiệm và lý thuyêt. }\end{array}$ \\
\hline 3 & $\begin{array}{l}\text { Người tính phải chọn bộ tham số tối } \\
\text { ưu }\end{array}$ & Tự động lọc ra bộ tham số tối ưu. \\
\hline 4 & $\begin{array}{l}\text { Người tính cần có chuyên môn sâu và } \\
\text { nhiều kinh nghiệm. }\end{array}$ & $\begin{array}{l}\text { Người tính không cần có chuyên } \\
\text { môn sâu và nhiều kinh nghiệm. }\end{array}$ \\
\hline
\end{tabular}

\section{Kết luận}

Bài báo đã trình bày chi tiết, đầy đủ về cơ sở lý thuyết phương pháp xác định các tham số của hàm hiệp phương sai lý thuyết từ các giá trị của hàm hiệp phương sai thực nghiệm trong bài toán Collocation bình phương nhỏ nhất.
Chương trình xác định các tham số tối ưu của hàm hiệp phương sai lý thuyết được xây dựng

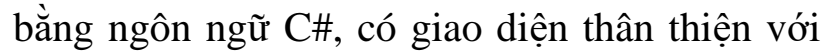
người dùng. Chương trình có tên là "Fitting Covariance Function". Kết quả của chương trình được so sánh với kết quả tính của "COVFIT” đã khẳng định tính chính xác của chương trình. 


\section{Nghiên cúru}

Chương trình "Fitting Covariance Function" có ưu điểm so với "COVFIT” là: Tự động tính toán, không cần nhập các tham số đầu vào; Tự động vẽ đồ thị của hiệp phương sai thực nghiệm và lý thuyết; Tự động lọc ra bộ tham số tối ưu; Người tính không cần có chuyên môn sâu và nhiều kinh nghiệm. Chương trình đã khắc phục được những hạn chế của chương trình "COVFIT" trong bộ phần mềm GRAVSOFT.O

\section{Lò̀i cảm ơn}

Các tác giả xin gửi lời cảm ơn đến Bộ Giáo dục và Đào tạo, Trường Đại học Mỏ - Địa chất đã hỗ trợ chúng tôi trong các nghiên cứu này thông qua đề tài cấp Bộ "Nghiên cứu đề xuất phương pháp xác định độ sâu đáy biển từ số liệu dị thường trọng lực trên khu vực Biển Đông" mã số: B2021-MDA-06.

\section{Tài liệu tham khảo}

[1]. Nguyễn Văn Sáng và các cộng sự (2020), "Marine gravity anomaly mapping for the Gulf of Tonkin area (Vietnam) using Cryosat2 and Saral/AltiKa satellite altimetry data", Advances in Space Research. 66(3), pp. 505519.

\section{Summary}

The optimized solution of theoretical covariance function in LSC method for determination of the parameters

Nguyen Thanh Le, Le Quy Don Technical University

Nguyen Van Sang, Hanoi University of Mining and Geology

Dinh Xuan Manh, Viet Nam Institude of Geodesy and Catography

This paper researchs to determine optimal parameters of theoretical covariance functions in LSC method. The theoretical basis of method had been studies in detail. On the theoretical basis, the program "Fitting Covariance Function" determines the optimal parameters of the theoretical covariance function built in $\mathrm{C \#}$ language. On the new program, the authors have experimentally calculated the data in the Central region, the optimal parameters obtained are: $\mathrm{N}=224 ; f=0,108$; $\mathrm{A}$ $=0,15.10^{6}$; Phương sai $=125,91 \mathrm{mGal}^{2} ; \mathrm{R}_{\mathrm{E}}-\mathrm{R}_{\mathrm{B}}=-0,471 \mathrm{~km}$. Received parameters were checked manually on the program "COVFIT" in GRAVSOFT software package to confirm the correctness. Compared to "COVFIT", the new program has the following advantages: Automatic calculation, no need to enter inputThe parameters; Automatic plotting of emprical and theoretical covariances; Automatic filters the optimal parameters; The calculator does not need deep expertise and a lot of experience. The program has overcome the limitations of the program "COVFIT".O 